\title{
Low-frequency oscillations and convective phenomena in a density-inverted vibrofluidized granular system
}

\author{
C. R. K. Windows-Yule, ${ }^{1}$ N. Rivas, ${ }^{2}$ D. J. Parker, ${ }^{1}$ and A. R. Thornton ${ }^{3}$ \\ ${ }^{1}$ School of Physics and Astronomy, University of Birmingham, Edgbaston, Birmingham B15 2TT, United Kingdom \\ ${ }^{2}$ Multi-Scale Mechanics (MSM), MESA+, CTW, University of Twente, PO Box 217, 7500 AE Enschede, The Netherlands \\ ${ }^{3}$ Multi-Scale Mechanics (MSM) and Mathematics of Computational Science (MaCS), MESA+, CTW, \\ University of Twente, PO Box 217, 7500 AE Enschede, The Netherlands
}

(Received 20 February 2014; revised manuscript received 27 August 2014; published 19 December 2014)

\begin{abstract}
Low-frequency oscillations (LFOs) are thought to play an important role in the transition between the Leidenfrost and convective states of a vibrated granular bed. This work details the experimental observation of LFOs, which are found to be consistently present for a range of driving frequencies and amplitudes, with particles of varying material and using containers of differing material properties. The experimentally acquired results show a close qualitative and quantitative agreement with both theory and simulations across the range of parameters tested. Strong agreement between experimental and simulation results was also observed when investigating the influence of sidewall dissipation on LFOs and vertical density profiles. This paper additionally provides evidence of two phenomena present in the Leidenfrost state: a circulatory motion over extended time periods in near-crystalline configurations, and a Leidenfrost-like state in which the dense upper region displays an unusual inverse thermal convection.
\end{abstract}

DOI: 10.1103/PhysRevE.90.062205

PACS number(s): 45.70.-n, 81.05.Rm

\section{INTRODUCTION}

Granular materials, large collections of discrete, macroscopic particles, have been the subject of considerable research for over two centuries, due largely to their ubiquity in nature and industry [1], and the plethora of interesting phenomena they exhibit [2]. They can exist in numerous states, many of which are analogous to those of a molecular material. Examples include: convection [3]; phase separation [4,5]; Faraday-like patterns [6]; and, the granular Leidenfrost state [7], wherein a dense region of grains is supported from below by a dilute, energetic gaseous region, in direct analogy to the eponymous state observed in classical fluids [8]. A simplified representation of a granular system in the Leidenfrost state may be seen in Fig. 1, with an experimentally acquired density profile corresponding to this state provided in Fig. 2. Although the vertically vibrated narrow box geometry has been extensively studied [9-12], recent simulation and theoretical work [13] has suggested the presence of a previously unobserved phenomenon: low-frequency oscillations. This term refers to the periodicity observed in the motion of the dense upper region of a granulate in a density-inverted state, i.e., in the Leidenfrost or convective regimes. The term LFO refers to the fact that the frequency of this oscillatory motion is considerably lower than that of the vibrating plate exciting the granular bed.

In the current work, we provide experimental evidence of the existence of LFOs in a three-dimensional, vibrofluidized granular bed. In addition to our confirmation of previous theoretical and simulation results, we also report the observation of two convective phenomena. The role of dissipation at the horizontal boundaries of the system is also explored; sidewalls have previously been shown to significantly affect the behavior of both two- [14] and three-dimensional [15] systems, and as such are likely to have a significant impact on the phenomena observed in the quasi-one-dimensional and laterally constrained three-dimensional systems such as those used here.
The paper is structured as follows: we begin by describing our basic experimental setup (Sec. II A), the manner in which data is extracted from the system (Sec. IIB), and how this setup is recreated in simulations (Sec. IIC), before briefly summarizing the continuum model introduced in Ref. [13] (Sec. II D). We then proceed to present our major results, first comparing our experimental findings to the predictions of the existing theoretical and simulation models (Secs. III A, III B), before presenting two convective phenomena (Secs. IIIC, IIID), and discussing the possible underlying mechanisms thereof. A summary of our major results and their implications is provided in Sec. IV.

\section{SYSTEM DETAILS}

\section{A. Experimental setup}

In order to experimentally observe the Leidenfrost state, a granular bed consisting of 12 square-packed layers of glass or steel spheres is housed in a cuboid container of dimensions $l_{x} \times l_{y} \times l_{z}=25 \times 25 \times 250 \mathrm{~mm}$ and vibrated sinusoidally in the vertical direction. A range of vibrational frequencies, $\omega \in(88,572)$, and peak accelerations, $A \in(0.8,6.0) \mathrm{mm}$, are used to excite the system. The significant variation in $\omega$ and $A$, and hence the dimensionless shaking strength, $S=\frac{\omega^{2} A^{2}}{g d}$, allows us to explore a variety of differing dynamical states.

The height $l_{z}$ of the container is adequate to ensure an effectively open system. Spheres of diameter $d=1,2,3$, and $5 \mathrm{~mm}$ are used, giving the system a range of dimensionless widths, $\tilde{l}_{x}=l_{x} / d \in(5,25)$. The lower limit of this range corresponds to the situation of a granular column, wherein horizontally inhomogeneous states are suppressed and, therefore, the system can be considered quasi-one-dimensional. This frustration of collective motion facilitates the clear observation of LFOs. The base and sidewalls of the container are steel, giving an effective particle-wall coefficient of restitution 


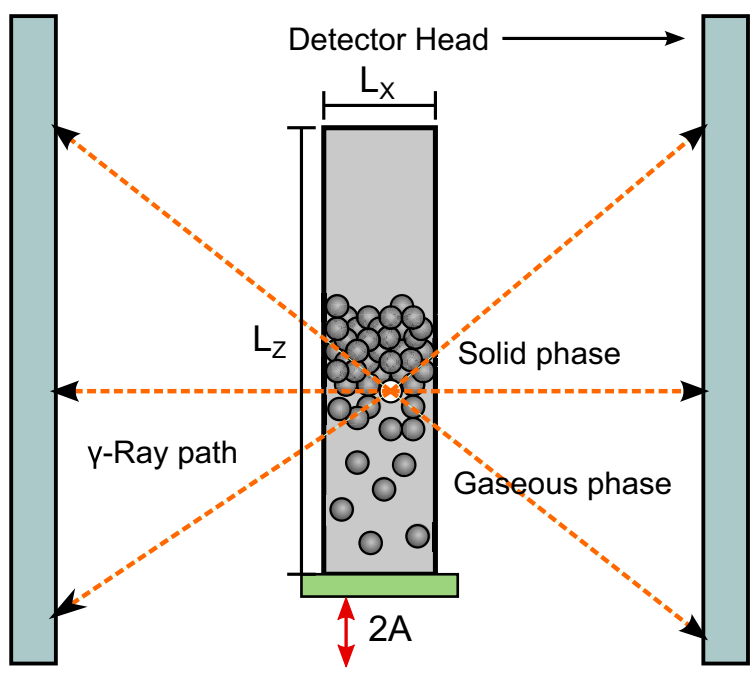

FIG. 1. (Color online) Schematic representation of the experimental system, showing a granular bed in the density-inverted Leidenfrost state. Although in reality the radioactive tracer particle is physically identical to all others in the system, for the sake of clarity this particle is here highlighted in white. Dotted orange arrows represent the paths taken by the $\gamma$ rays emitted by the tracer, while the solid red arrow represents the direction of vibration.

$\varepsilon_{w}=0.70 .{ }^{1}$ The use of a steel wall ensures rigidity, and also removes the possibility of static charges affecting the system. A single opposing pair of sidewalls can be replaced with perspex $\left(\varepsilon_{w}=0.33\right.$ ), allowing the effects of sidewall dissipation to be investigated.

\section{B. Positron emission particle tracking}

Experimental data is acquired using positron emission particle tracking (PEPT), a noninvasive technique whereby a

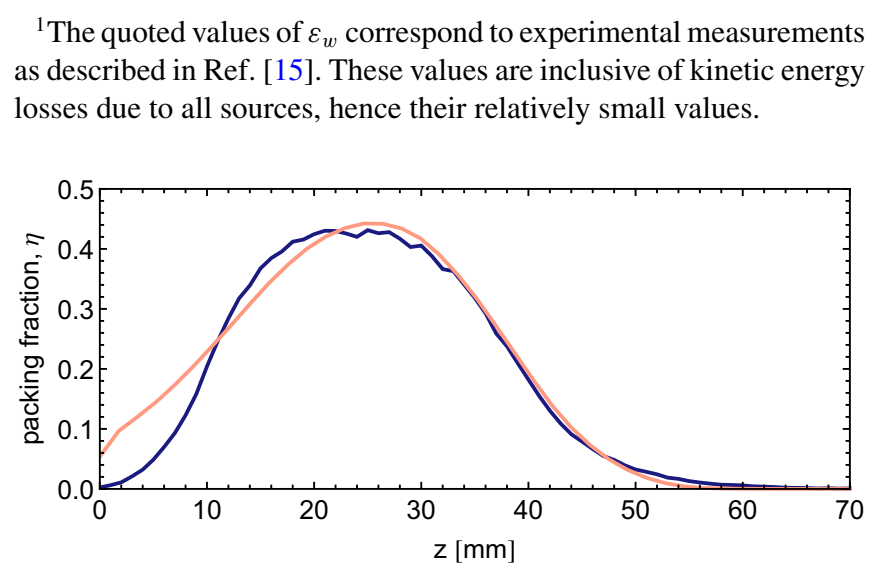

FIG. 2. (Color online) Experimentally (blue/dark gray) and simulation (red/light gray) acquired one-dimensional vertical density profile, demonstrating the general form expected for a granular bed in the density-inverted Leidenfrost state. In this image, we clearly see a particle-dense region resting atop a considerably more dilute, gaseous phase. The data presented correspond to a bed of $1 \mathrm{~mm}$ diameter glass particles excited via vibration at a constant frequency $\omega=157 \mathrm{~Hz}$ and amplitude $A=4 \mathrm{~mm}$. single particle, physically identical to the others in the system, is labeled with a $\beta^{+}$-emitting isotope. The back-to-back pairs of $\gamma$ rays emitted due to the rapid annihilation of positrons with electrons within this tracer particle are detected using a dual-headed $\gamma$ camera, and can be used to triangulate its position with millimetre precision and millisecond time resolution [16]. A simple illustration of this process may be seen in Fig. 1. The time-averaged motion of this single particle can then be used to give information pertaining to the system as a whole. For instance, in PEPT data may be used to create onedimensional density profiles. This is achieved by subdividing the computational volume into a series of thin horizontal segments, each of height $d z$. The fraction of time spent by the tracer in each of these segments may then be recorded. Due to the ergodicity of the system under investigation, this residence time fraction is directly proportional to the local packing density within each region allowing, for adequately small segments, a vertical density profile to be reproduced. Examples of such profiles may be seen in Figs. 2 and 6.

In order to ensure reliable statistics, all experiments are conducted over a period of between 45-120 min, dependent on the density of the system under investigation and the strength with which it is driven. Since denser and/or more weakly excited systems exhibit slower dynamics, the run duration must be accordingly increased in order to allow the tracer particle to fully explore the entire system, including both the dense and dilute phases. For further information regarding PEPT, see Refs. [16,17].

\section{Event-driven simulations}

Simulations are performed using an event-driven (ED) molecular dynamics [18]. The algorithm uses a hard-sphere model, assuming binary collisions with no overlap and no longrange forces between particles. Collisions between particles are modeled by normal and tangential velocity-dependent restitution coefficients, following the expression in Ref. [19]. This is the same simulation code as used in Ref. [13], where a more detailed description of the algorithm can be found. Material properties were chosen such that, at a typical particle velocity $v_{0}=0.3 \mathrm{~ms}^{-1}$, the relevant coefficients of restitution, $r_{0}$, are $r_{0} \sim 0.90$ and $r_{o} \sim 0.95$ for glass and steel particles respectively. The use of a velocity-dependent $r_{0}$ ensures that dissipation is not overestimated at high particle densities, as can occur when using constant coefficients [20]. Static and dynamic friction coefficients ( $\mu_{s}$ and $\mu_{d}$, respectively) are also considered, and held constant at $\mu_{s}=\mu_{d}=0.05$. For particle-wall collisions the value $r_{0}^{w}=0.7$ was used for glass, and $r_{0}^{w}=0.75$ for steel particles, while $\mu_{s}^{w}=\mu_{d}^{w}=0.15$. Although it was verified that these values are not critical to the observation of the phenomena, the system is quantitatively sensitive to them. Experimental values are used where known.

\section{Continuum model}

The frequency of the LFOs is also theoretically predicted by a continuum model (described in detail in Ref. [13]), where the only input parameter is the experimentally measured vertical density profile. In this model, a granulate in the Leidenfrost state is considered to have two distinct phases: a dense, solidlike region of density $\rho_{s}$ and mass per unit area in the 

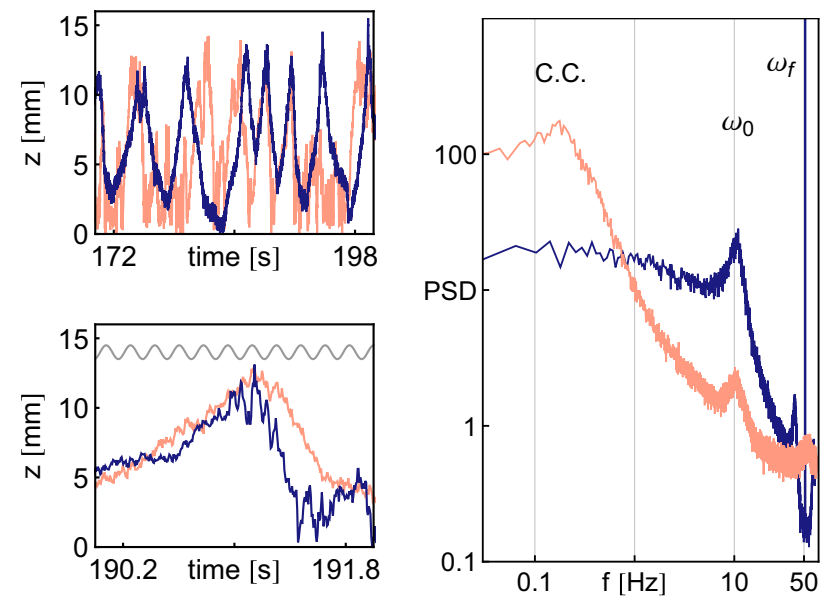

FIG. 3. (Color online) (a) Vertical position, $z(t)$, of a single particle for experiment (blue/dark gray) and simulation (red/light gray), for a system of 3-mm glass spheres with $\omega=327 \mathrm{~Hz}$ and $A=2 \mathrm{~mm}$. Data is shown for larger (above) and smaller (below) timescales. (b) Power spectra of $z(t)$, for experimental (blue, dark gray) and simulation (red, light gray) data, showing $\omega_{f}$ and $\omega_{0}$, the driving and LFO frequency respectively, as well as the crystalline convection frequency, C.C.

horizontal plane, $m_{s}$, and a gaseous region of density $\rho_{g}$. The periodic vertical motion of the dense region can be described as a forced harmonic oscillator, allowing the frequency of LFOs, $\omega_{0}$, to be simply calculated as:

$$
\omega_{0}=\sqrt{\frac{g \rho_{g}}{m_{s}}} .
$$

\section{RESULTS AND ANALYSIS}

\section{A. Experimental observation of LFOs}

Although the existence of LFOs in density inverted systems has been shown in simulations and predicted theoretically in Ref. [13], their presence has not been confirmed experimentally. ${ }^{2}$ Figure 3 shows a typical example of the evolution of the tracer particle's vertical position, $z(t)$, for both experimental and simulation data, alongside corresponding fast Fourier transforms (FFTs). To minimize noise, $z(t)$ is split into a series of 10 -s traces, each containing approximately $3 \times 10^{4}$ data points, and the corresponding FFTs averaged. The presence of LFOs can be identified by a broad peak in the power spectrum at a frequency $\omega_{0}$ one or two orders of magnitude lower than the driving frequency $\omega_{f}$. Thus, the existence of a clearly defined low-frequency peak in Fig. 3(b) evidences the existence of LFOs in an experimental system. It should be noted that Fig. 3 is indeed a typical example: LFOs are observed over a wide range of driving frequencies, $\omega_{f} \in(94,503) \mathrm{Hz}$, and amplitudes, $A \in(1,5) \mathrm{mm}$ (the upper values being limited by the maximum obtainable velocity of the shaker used to

\footnotetext{
${ }^{2}$ Behavior suggestive of the presence of LFOs may be observed in the data of Folli et al. [31]. However, due to considerable disparities between their system and the current setup, it is not possible to confirm that this is the same phenomenon.
}
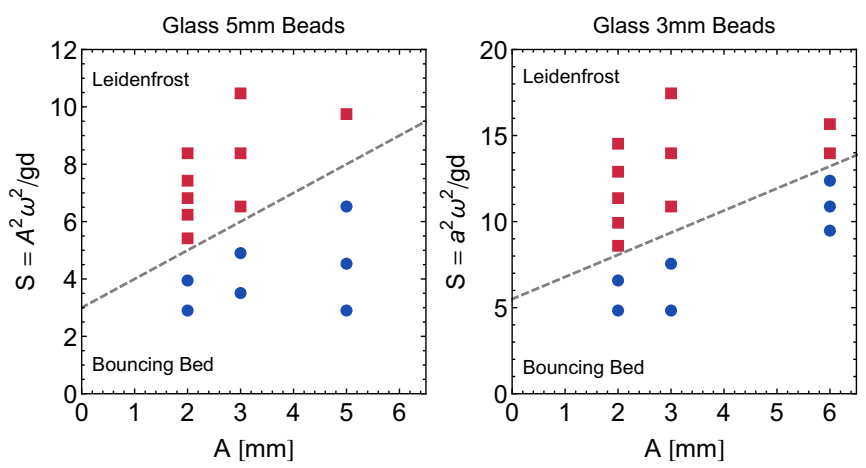

FIG. 4. (Color online) Phase space for 5-mm (left) and 3-mm (right) glass beads. As the magnitude of the energy injection, $S$, increases, the system goes from the bouncing bed (blue dots) to the Leidenfrost (red squares) state. The transition region is roughly delimited by the horizontal dashed gray line.

drive the system). Monodisperse beds composed of both steel and glass particles also exhibit LFOs over a range of particle sizes $d \in(2,5) \mathrm{mm}$, and for both steel- and acrylic-walled systems, showing it to be a robust and easily reproducible phenomenon. In the following discussion, we initially focus on glass beads of $3 \mathrm{~mm}$ and $5 \mathrm{~mm}$ diameter, in order to avoid the presence of buoyancy-driven convection, which typically dominates the system dynamics for smaller particle sizes. Figure 4 shows data in the $S$ - $A$ phase space for these two cases. Remarkably, all experimental data sets for which the system exists in the Leidenfrost state are seen to exhibit low-frequency oscillations. Thus, the phase diagrams presented provide a clear indication of the ranges of parameters for which one may expect to observe LFOs.

From the FFTs of $z(t)$ we are able to obtain the dominant LFO frequency. Figure 5 shows $\omega_{0}$ for experiments, simulations, and the value computed from the model. A good agreement is observed between three cases, in the case of the model increasing with the energy input. This is to be expected, as the model is formally only valid in the large shaking amplitude limit [13]. For all other particle numbers and sizes, wall and particle inelasticities and driving parameters explored, the experimentally obtained values of $\omega_{0}$ agree with those produced by both the ED and continuum models to within $10 \%$. The persistent presence of LFOs across such a broad range of parameter space, including the highly elastic
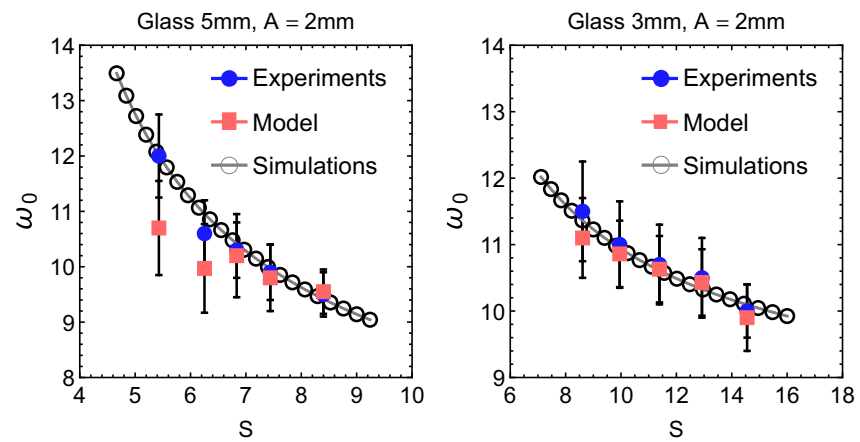

FIG. 5. (Color online) Experimental and theoretical values of $\omega_{0}$ as a function of the shaking strength $S=A^{2} \omega_{f}^{2} / g d$. Data for $d=$ $3 \mathrm{~mm}$ and $d=5 \mathrm{~mm}$ glass particles, for $A=2 \mathrm{~mm}$. 


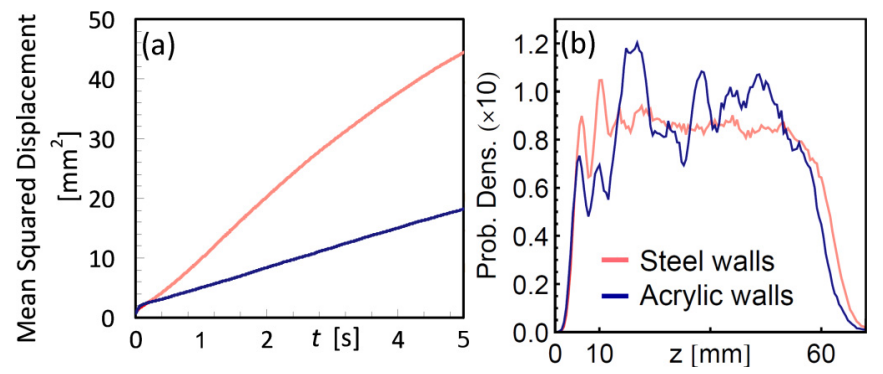

FIG. 6. (Color online) (a) Experimental mean-squared displacement, $M(t)$, for particles in the dense upper region of the system for steel (blue/dark gray) and acrylic (red/light gray) sidewalls. For both cases, $\omega=327 \mathrm{~Hz}, A=2 \mathrm{~mm}$, and $l_{x}=5$. (b) Normalized vertical packing profiles corresponding to the same system parameters.

limit at which the behavior of granular systems approaches that of classical, molecular systems [21], suggests that an analogous phenomenon may also be present in classical fluids.

\section{B. Effect of sidewall dissipation}

In a constrained system, such as the one described here, we expect the dissipation of the walls to significantly affect the dynamics of the bed. Thus, to further explore the generality of our results, the material of a single pair of sidewalls is altered, hence altering $r_{0}^{w}$. Notice that, even in constrained systems, if a system's packing fraction is large, $r_{0}^{w}$ is typically considered unimportant. Thus, the current setup allows us to explore the competing effects due to lateral confinement and packing density. Comparison of data acquired from otherwise identical systems with steel $\left(\varepsilon_{w}=0.7\right)$ and acrylic $\left(\varepsilon_{w}=0.33\right)$ sidewalls shows the increased dissipation caused by the less elastic sidewalls to considerably affect even highly dense systems, even with all other parameters held constant. This observation is illustrated by the packing density distributions shown in Fig. 6(b). It should be noted, however, that LFOs are still found to be present for both sidewall materials. It is additionally worth specifically noting that we do not expect the inclusion of the acrylic walls to introduce any appreciable effects due to triboelectric charging, due both to the relatively large sizes and masses of the particles concerned, as well as the frequent collisions experienced by particles and the system's two remaining steel sidewalls and base, which will act to rapidly dissipate charge. In both of the profiles presented in Fig. 6(b), we see the existence of multiple peaks and valleys, indicative of the presence of crystalline structure within the bed. However, these local maxima and minima are considerably more pronounced in the acrylic-walled system than in its steel-walled counterpart, indicating a significantly increased degree of crystallization in the former. This makes sense, as higher wall dissipation will clearly lead to a decrease in the fluctuating component of the kinetic energy (granular temperature). Figure 6(a) shows the typical mean-squared displacement of particles in the upper region of the system for both sidewall types. The markedly reduced mobility of particles in the acrylic-walled system again demonstrates the heat-sink-like effect of the dissipative system boundaries.

It is interesting to note the marked differences between the density profiles presented in Fig. 6(a) and that shown in Fig. 2, another clear demonstration that LFOs exist for a variety of system parameters and dynamic states, and also coexist with various other phenomena.

\section{Crystalline convection in the Leidenfrost state}

Analysis of $z(t)$ and the corresponding power spectra for denser, less-fluidized systems shows evidence of an extremely slow, pseudoperiodic motion, with a frequency approximately two orders of magnitude lower even than the previously discussed LFOs [see Figs. 3(a) and 3(b)]. This slow migration of particles was also observed in simulations, as illustrated in Fig. 3(a). However, in order to obtain the periodic behavior observed in experiments, it was necessary to modify the frictional and elastic coefficients of the collision model for particle-wall interactions, indicating a strong influence of the side boundaries on this phenomenon. Although a somewhat similar phenomenon has been previously reported in a multiphase system [29], we observe such behavior in a monodisperse granular bed. It is possible, on an adequately long time scale $-O\left(10^{3}\right)$ s- to observe dynamics reminiscent of convective motion within the densely packed crystalline phase of the upper region. Specifically, grains are seen to move, on average, upward in the central region of the domain and downward in the vicinity of the lateral boundaries. This motion is distinct from the conventional, continuous convective flow previously observed [30]. Here, the bed maintains a crystalline structure, with the migration of particles occurring in sudden, discrete motions separated by periods of inactivity. The mean flow rate associated with this crystalline convection is typically $O(0.1) \mathrm{mm} / \mathrm{s}$, compared to the values of $\approx 3 \mathrm{~mm} / \mathrm{s}$ for reverse convection and $\approx 6 \mathrm{~mm} / \mathrm{s}$ typical of the normal convection observed within the system. Further study of this state may give insight into the origins of the transition of a granular system between a mechanically stable solidlike state and a disordered, fluidlike state, a matter on which there is no general consensus, despite significant research in the area [32]. Such research could also lead to an improved understanding of the Leidenfrost-convection transition.

\section{Reverse convection in the Leidenfrost state}

In a system such as the one detailed here, a granular bed may exhibit thermal or buoyancy-driven convection [3]. In systems with dissipative side boundaries, one may observe a wall-enhanced thermal convection, whereby a region of increased density near the sidewalls leads to the formation of a pair of convection rolls reminiscent of Rayleigh-Bénard cells [22]; the rolls move downward in a thin stream near the walls, where increased dissipation leads to a locally higher density and lower energy, and upward in the center of the container where the density is, accordingly, reduced [23]. It should be noted that this form of convection is distinct from the frictionally driven convection observed in less strongly excited systems than those discussed here [24]. For data sets where $\tilde{l}_{x}>5$, such wall-induced convection is indeed observed. However, for certain combinations of $\omega$ and $A$, with $\tilde{l}_{x}=25$, one observes a density-inverted state in which the denser upper region displays convective motion whose sense is opposite to the expected (see Fig. 7). Previously, reverse convection had only been observed in frictionally driven systems [25]; 

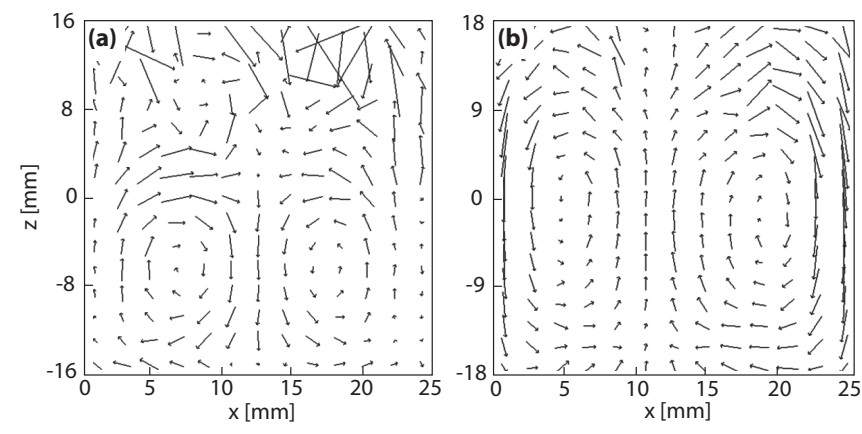

FIG. 7. (a) Velocity field for the dense upper region of a system of 1-mm particles in a density-inverted state, driven at $A=4 \mathrm{~mm}, \omega=$ $157 \mathrm{~Hz}$. The arrows, whose lengths and orientations are representative of the magnitude and direction of the average velocity through a section of the field, show a net downward motion in the center of the system and a net upward motion near the side boundaries. (b) Velocity plot showing, for comparison, typical wall-enhanced convection. The driving parameters for this case are $A=1 \mathrm{~mm}$ and $\omega=509 \mathrm{~Hz}$.

here, we observe the inversion of buoyancy-driven convection in experimental systems. We propose a tentative explanation for this effect: sidewall dissipation is more influential in lower-density regions [26], where the ratio of particle-wall to particle-particle collisions is higher. Thus, in the dilute lower region of the Leidenfrost state, a pronounced increase in density and decrease in temperature will occur near the walls of the system, leading to a decreased pressure in this region [27]. Conversely, for the upper region of the bed, one observes an increased relative density in the central region of the bed (see Fig. 8). This greater compaction near the center may be due to the increased pressure acting on the upper bed due to the relatively energetic gas in the central region below. At the interface between the upper and lower regions of the bed, collisions from particles in the energetic lower region will break the structure of particles in the dense region, creating a localized volume of more energetic, lower-density particles, which will tend to rise through the bed. These relatively mobile particles are likely to be pushed radially outward from the center of the container due to the pressure gradients in the dilute region below, increasing the probability that upward motion will occur near the side boundaries of the container. Moreover, the slightly decreased density in the upper bed near the sidewalls means that the upward transit of the energetic particles is less likely to be impeded. Due to conservation of mass flux, this will naturally result in a pair of inversely oriented convection rolls within the system. The combination of $\omega$ and $A$ at which this phenomenon is observed suggests that it may be representative of a transitional state between the Leidenfrost state and the Faraday wave state [28]. It should be noted that the behavior detailed above was
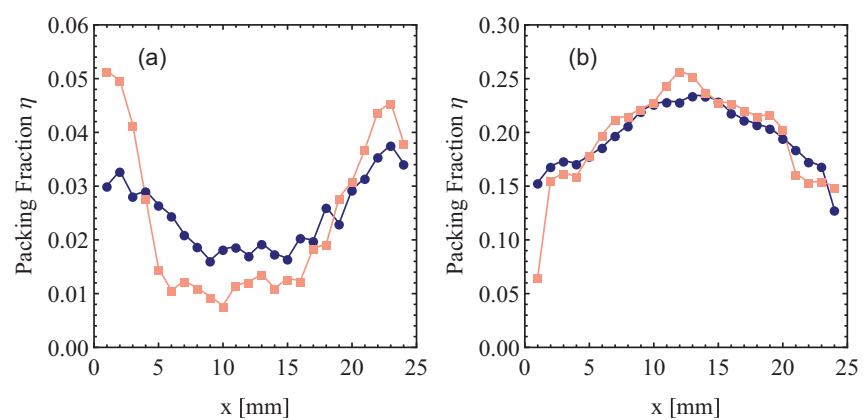

FIG. 8. (Color online) Packing fraction, $\eta$ as a function of horizontal position for (a) the lower, gaseous region of the system and (b) the dense upper region, for steel (blue, dark gray) and acrylic (red, light gray) sidewalls.

found to be reproducible, and was still observed when the system's sidewall material was altered, although differences in the convective flow rate and the specific densities of each phase were observed for the two cases. Specifically, as the sidewall coefficient of restitution was decreased from 0.70 to 0.33 , the convective flow rate of the system was found to increase from $\approx 2.5 \mathrm{~mm} / \mathrm{s}$ to $\approx 3.7 \mathrm{~mm} / \mathrm{s}$. The authors believe this phenomenon to be worthy of further study.

\section{CONCLUSIONS}

Analysis of the motion of a single particle in a granular bed, acquired using positron emission particle tracking, has been used to provide strong experimental evidence of low-frequency oscillations in density-inverted, vibrofluidized granular systems. The experimentally observed frequencies of these oscillations, and the variation of these frequencies with numerous system parameters, were found to correspond closely to simulation and continuum theory. The observation of LFOs over a wide range of parameters supports the hypothesis that they are a fundamental feature of the Leidenfrost state, meaning that future study of these oscillations could prove crucial to the understanding of the transition of a granular system between the Leidenfrost and convective regimes. The findings of this study also suggest the existence of two convective phenomena, the further study of which may provide valuable insight into transitions involving the Leidenfrost state.

\section{ACKNOWLEDGMENTS}

The authors wish to acknowledge Stefan Luding for useful discussions. This work was financially supported by the NWO-STW VICI Grant No. 10828 and the Hawkesworth Scholarship.
[1] H. M. Jaeger, S. R. Nagel, and R. P. Behringer, Phys. Today 49(4), 32 (1996).

[2] K. van der Weele, Contemp. Phys. 49, 157 (2008).

[3] R. Ramirez, D. Risso, and P. Cordero, Phys. Rev. Lett. 85, 1230 (2000).
[4] J. S. Olafsen and J. S. Urbach, Phys. Rev. Lett. 81, 4369 (1998).

[5] P. Melby, F. Vega Reyes, A. Prevost, R. Robertson, P. Kumar, D. A. Egolf, and J. S. Urbach, J. Phys.: Condens. Matter 17, S2689 (2005). 
[6] S. Fauve, S. Douady, and C. Laroche, J. Phys. (Paris) 50, Suppl. 3, 187 (1989).

[7] B. Meerson, T. Pöschel, and Y. Bromberg, Phys. Rev. Lett. 91, 024301 (2003).

[8] J. G. Leidenfrost, Int. J. Heat Mass Transfer 9, 1153 (1966).

[9] B. Thomas, M. O. Mason, Y. A. Liu, and A. M. Squires, Powder Technology 57, 267 (1989).

[10] P. Eshuis, K. van der Weele, D. van der Meer, and D. Lohse, Phys. Rev. Lett. 95, 258001 (2005).

[11] P. Eshuis, K. van der Weele, D. van der Meer, R. Bos, and D. Lohse, Phys. Fluids 19, 123301 (2007).

[12] Lim EWC, Eur. Phys. J. E. 32, 365 (2010).

[13] N. Rivas, S. Luding, and A. R. Thornton, NJP 15, 113043 (2013).

[14] J. S. van Zon, J. Kreft, D. I. Goldman, D. Miracle, J. B. Swift, and H. L. Swinney, Phys. Rev. E 70, 040301(R) (2004).

[15] C. R. K. Windows-Yule, N. Rivas, and D. J. Parker, Phys. Rev. Lett. 111, 038001 (2013).

[16] D. J. Parker, R. N. Forster, P. Fowles, and P. S. Takhar, Nucl. Instrum. Methods Phys. Res., Sect. A 477, 540 (2002).

[17] R. D. Wildman, J. M. Huntley, J.-P. Hansen, D. J. Parker, and D. A. Allen, Phys. Rev. E 62, 3826 (2000).

[18] B. D. Lubachevsky, J. Comput. Phys. 94, 255 (1991).
[19] S. McNamara and E. Falcon, Phys. Rev. E 71, 031302 (2005).

[20] T. Poschel, N. V. Brilliantov, and T. Schwager, Physica A 325, 274 (2003).

[21] C. Lun, S. B. Savage, D. J. Jeffrey, and N. Cherpurniy, J. Fluid Mech. 140, 223 (1984).

[22] K. M. Aoki, T. Akiyama, Y. Maki, and T. Watanabe, Phys. Rev. E 54, 874 (1996).

[23] J. Talbot and P. Viot, Phys. Rev. Lett. 89, 064301 (2002).

[24] J. B. Knight, Phys. Rev. E 55, 6016 (1997).

[25] J. B. Knight, H. M. Jaeger, and S. R. Nagel, Phys. Rev. Lett. 70, 3728 (1993).

[26] R. D. Wildman, J. M. Huntley, and D. J. Parker, Phys. Rev. E 63, 061311 (2001).

[27] I. Goldhirsch and G. Zanetti, Phys. Rev. Lett. 70, 1619 (1993).

[28] J. A. Carrillo, T. Pöschel, and C. Saluena, J. Fluid Mech. 597, 119 (2008).

[29] J. A. C. Gallas, H. J. Herrmann, T. Pöschel, and S. Sokolowski, J. Stat. Phys. 82, 443 (1996).

[30] S. McNamara and S. Luding, Phys. Rev. E 58, 813 (1998).

[31] V. Folli, N. Ghofraniha, A. Puglisi, L. Leuzzi, and C. Conti, Sci. Rep. 3, 2251 (2013).

[32] T. S. Majmudar, M. Sperl, S. Luding, and R. P. Behringer, Phys. Rev. Lett. 98, 058001 (2007). 